INVESTIGACIÓN

\title{
EQUIDAD SOCIAL: UN RETO POR RECORRER PARA LA SOCIEDAD COLOMBIANA*
}

\section{SOCIAL EOUITY: A CHALLENGE FOR THE COLOMBIAN SOCIETY}

\author{
Isabel Lagunes** \\ Ismael Hernández ${ }^{\star \star *}$
}

Recibido: 20 de noviembre de 2017

Evaluado: 01 de diciembre de 2017

Aprobado: 2 de febrero de 2018

\section{RESUMEN}

El objetivo de esta investigación es entender cómo el avance científico y las tendencias culturales de la actualidad se alejan de la racionalidad y de la búsqueda de la verdadera equidad social, al contrario, sugieren ligar la calidad de vida a la propensión marginal del consumo. Es así como se calcula en los principales indicadores en el mundo al respecto -el coeficiente de Gini, el índice Theil y el índice Palma-. La prioridad a la medición de los ingresos de los ciudadanos para calcular la inequidad, implica enfocar políticas y soluciones netamente monetarias que, vistos de forma directa, conllevan al simple incentivo del consumo sin tener repercusión de fondo en el desarrollo social o en la calidad de vida de los seres humanos. La implementación de la medición tomando el método que sugiere Índice de Pobreza Multidimensional adaptado para Colombia a partir del año 2010, fue el insumo para la implementación de políticas sociales, que permitieron corregir las carencias de los ciudadanos objetivo y, como consecuencia, en el mediano plazo afectan de forma positiva el comportamiento de los indicadores monetarios. Teniendo en cuenta

* Artículo de investigación.

** Candidata a Doctora de Gerencia y Política Social, Universidad de Baja California. Correo electrónico: ilagunes@msev.gob.mx, Número ORCID orcid.org/0000-0002-4631-4385

*** Candidato a Doctor de Gerencia y Política Social, Universidad de Baja California. Correo electrónico: ihernandezr@unal.edu.co Número ORCID orcid.org/0000-0002-4183-9879 
los datos disponibles en el Departamento Nacional de Estadística se realizará un comparativo entre la evolución del coeficiente de Gini, el Índice de Pobreza Monetaria entre el año 2000 hasta el año 2015 y el histórico reflejado en el Índice de pobreza Multidimensional a partir del año 2010. Palabras clave: índice de pobreza social, Colombia.

\begin{abstract}
The interest of this reflection is to understand how the scientific advance and the cultural tendencies of the present time get away of the rationality and the search of the true social equality, on the contrary suggests to tie the quality of life to the marginal propensity to the consumption, as calculates the main indicators in the world, -The Gini coefficient, Theil index and the Palm index-. The priority of measuring citizens' incomes in order to calculate inequity is to focus purely monetary policies and solutions, which, when viewed directly, ultimately lead to the simple incentive of consumption without having a profound impact on social development or quality of life of human beings, the implementation of the methods that take the method that suggests multidimensional poverty index adapted for Colombia from the year 2010, was the input for the implementation of social policies, allowed to correct the shortcomings of the target citizens and as a consequence, in the medium term positively affect the behavior of the monetary indicators. Taking into account the data available in the National Department of Statistics, a comparison is made between the evolution of the Gini coefficient, the monetary poverty index between 2000 and 2015, and the historical reflection in the Multidimensional Poverty Index year 2010.
\end{abstract}

Keywords: Social Poverty Index, Colombia.

\section{INTRODUCCIÓN}

Es función del Estado intervenir en la producción de servicios que permitan el desarrollo de las personas, como parte de la sociedad. Sin embargo, un pueblo que haya logrado desarroIlar individualmente sus competencias de desempeño, es un pueblo con perfil emprendedor, claro está, las políticas públicas deben estar enfocadas para este efecto, lo describe claramente Zerda Álvaro (2009):

La construcción de un nuevo modelo económico implica una fuerte reorientación de la inversión pública desde el gasto improductivo e incluso destructivo (la guerra) hacia las actividades de apoyo al desarrollo con equidad 
de las fuerzas productivas, como educación, salud, investigación, innovación e infraestructura, que al mismo tiempo absorben mano de obra para reactivar la generación de empleo en una primera instancia. (p. 54).

El efecto positivo como resultado de la aplicación de estas políticas trasciende en la macroeconomía del Estado. Permite incorporar al sector productivo en esa franja poblacional etiquetada anteriormente como pobres, a la efectividad de la ejecución del gasto en las regiones y al compromiso pleno de cada ciudadano.

La naturaleza primordial de los Estados es su población y su compromiso debe ser la búsqueda de respuestas a las necesidades básicas de los ciudadanos. En la primera década del siglo actual, el gobierno nacional colombiano utilizó como base para identificar la población en estado de pobreza únicamente el Índice de Pobreza Monetaria, situación que generó varios acercamientos a la atención social. Esto fue el resultado de análisis realizados por tecnócratas que generaron programas de atención, con el fin de prestar servicios de salud, educación, servicios básicos y atención directa de forma monetaria entre otros, estos programas no han logrado una distribución equitativa y no se ha marcado una etiqueta que se identifique con plena transparencia, este efecto lo expone claramente Carvajal German (2012):
Excelencia en el servicio y corrección en el gasto de dinero público son dos premisas de orden ético que, en principio, rigen para toda institución estatal. La naturaleza de este régimen no es ontológica, es deontológica, lo cual significa que su cumplimiento depende de la voluntad de los individuos (p. 37).

La corrupción es un comportamiento humano que puede reducirse con el repudio masivo de una sociedad que logre educarse bajo principios éticos, esta situación es posible sólo proporcionando a la sociedad las herramientas mínimas para lograr su desarrollo, elementos que van más allá del simple sustento o de la satisfacción del deseo por consumir. En este sentido, el modelo utilizado como Índice de Pobreza Multidimensional, para Colombia, determina una estructura para la identificación con un método científico de la población más vulnerable y su capacidad para acceder a estos derechos.

Es pertinente para la sociedad enfocar un modelo ideal de vida, posible de lograr, optando por preferir el bienestar general a lo personal y tener estos principios especialmente cuando se es funcionario del Estado. La corrupción se genera por una convicción contraria a lo expuesto anteriormente. Uno de los problemas que predominan en las instituciones actualmente, es viable erradicarlo a través de una educación 
general donde el comportamiento colectivo no permita la aceptación de ciudadanos con esta concepción.

El objetivo de esta investigación es indagar cómo afecta la aplicación de las políticas sociales, basados en el insumo propuesto por el Índice de Pobreza Multidimensional en Colombia a partir del año 2010, en los resultados y la evolución de los indicadores del Índice de Pobreza Monetaria.

Comprobar mediante cruce de la información disponible con metadatos del DANE, si los resultados y la tendencia en el Índice de Desigualdad -coeficiente de Gini- es coherente a los resultados. Igualmente, verificar mediante la información disponible en el DANE si los resultados de los indicadores, basados en ingresos, actúan como consecuencia en la aplicación de múltiples políticas sociales que proporcionen las herramientas a los individuos para su desarrollo.

\section{¿CÓMO SE DEFINE LA POBREZA?}

Las diferentes formas de medir la pobreza a nivel mundial basan sus indicadores en el ingreso de las personas, como lo sugiere el Banco Mundial "La línea de pobreza de 1 dólar por día", el valor utilizado corresponde al promedio de las líneas nacionales de pobreza adoptadas por los países con los menores niveles de ingreso per cápita en el mundo -cálculo de 1991, para 2005 se definió un umbral en US 1,25- en el caso de índice de Gini. Este indicador es una medida de concentración y permite calcular cualquier distribución de frecuencias. Sin embargo, en este ámbito de estudio, se utiliza para medir la igualdad o desigualdad referidas a la riqueza o renta de un país. En este aspecto Amartya Sen ha señalado que los principios éticos bien fundados suponen la igualdad entre los individuos, pero como la habilidad para aprovechar la igualdad de oportunidades varía con cada persona, el problema de la distribución de bienestar nunca podrá resolverse del todo.

\section{¿QUÉ ES EL IPM?}

El Índice de Pobreza Multidimensional (IPM), desarrollado por el Oxford Poverty \& Human Development Initiative (OPHI), es un indicador que refleja el grado de privación de las personas en un conjunto de dimensiones. La medida permite determinar la naturaleza de la privación -de acuerdo con las dimensiones seleccionadas - y la intensidad de la misma. El IPM es la combinación del porcentaje de personas consideradas pobres y de la proporción de dimensiones en las cuales los hogares son, en promedio, pobres.

La propuesta de IPM desarrollada por el Departamento Nacional de Planeación para Colombia está conformada por cinco dimensiones y quince variables. 
El IPM se desarrolla a través de la construcción una matriz de 1 o 0 , donde el 1 representa privación y 0 no privación. En la matriz (1) las filas representan los hogares y las columnas las quince variables evaluadas. Una vez establecidas las ponderaciones se realiza una suma ponderada para cada hogar y se determina si es pobre de acuerdo al umbral establecido, el umbral corresponde a que el promedio ponderado de las privaciones sea igual o superior a (5/15). En Colombia, las variables se dividen en dimensiones - cada dimensión pesa lo mismo-, y al interior de cada una de ellas, las variables toman el mismo peso. Las dimensiones y variables consideras para el IPM de Colombia son:

1. Condiciones educativas del hogar: logro educativo y analfabetismo.

2. Condiciones de la niñez y juventud: asistencia escolar, rezago escolar, acceso a servicios para el cuidado de la primera infancia y trabajo infantil.
3. Trabajo: desempleo de larga duración y empleo formal.

4. Salud: aseguramiento en salud y acceso a servicio de salud dada una necesidad.

5. Servicios públicos domiciliarios y condiciones de la vivienda: acceso a fuente de agua mejorada, eliminación de excretas, pisos, paredes exteriores y hacinamiento crítico.

\section{EVOLUCIÓN DEL IPM EN COLOMBIA}

A partir del año 2010 este indicador se ha calculado con regularidad, encontrando una reducción en doce puntos porcentuales de la pobreza en Colombia. Estos cálculos se basaron en la evolución de los indicadores de cada una de las dimensiones que sustenta el efecto positivo y la necesidad de dar continuidad y reforzar las políticas sociales que permiten el logro del objetivo.

Tabla 1. Evolución Índice de Pobreza Multidimensional en Colombia.

\begin{tabular}{|l|l|l|l|l|l|l|l|}
\hline \multirow{2}{*}{ \% personas pobres } & $\mathbf{2 0 1 0}$ & $\mathbf{2 0 1 1}$ & $\mathbf{2 0 1 2}$ & $\mathbf{2 0 1 3}$ & $\mathbf{2 0 1 4}$ & $\mathbf{2 0 1 5}$ & $\mathbf{2 0 1 6}$ \\
\cline { 2 - 8 } & 30,4 & 29,4 & 27 & 24,8 & 21,9 & 20,2 & 17,8 \\
\hline
\end{tabular}

Fuente: elaboración propia a partir de los cálculos Departamento Nacional de Planeación con base en ENCV 2002-2008 y DANE 2010-2016.

\section{ÍNDICE DE POBREZA MONETARIA}

El cálculo lo realiza el Departamento Nacional de Estadística junto con el Departamento
Nacional de Planeación desde una perspectiva multidimensional que consiste en medir la pobreza de acuerdo al ingreso de las personas. 
Tabla 2. Evolución Índice de pobreza monetaria en Colombia.

\begin{tabular}{|c|c|c|c|c|c|c|c|c|c|c|c|c|c|c|c|}
\hline \multirow{2}{*}{ 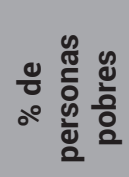 } & స్ & ஜ̊̀ે & ষ্ণ & นి & ஜั & 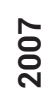 & రి & ᄋ్ & 음 & $\overline{\text { న }}$ & 畓 & $\stackrel{m}{\frac{m}{N}}$ & $\frac{\pi}{\text { Na }}$ & $\frac{10}{2}$ & $\stackrel{\circ}{\text { N }}$ \\
\hline & 50 & 49,7 & 49,8 & 48 & 47,4 & 45 & 42 & 40,3 & 37,2 & 34,1 & 32,7 & 30,6 & 28,5 & 28 & 27,8 \\
\hline
\end{tabular}

Fuente: elaboración propia a partir de información del Departamento Nacional de Estadística 2016.

\section{DESIGUALDAD SOCIAL}

La interacción entre crecimiento y desigualdad no necesariamente tienen una relación directa, la dirección de una o de la otra no están enfocadas implícitamente en el mismo sentido. Así surge la hipótesis de Kuznets, que formula la existencia de una relación directa entre el crecimiento y la desigualdad en las primeras etapas del desarrollo de un país, hasta llegar a un punto crítico en el cual la desigualdad se reduce y la economía se incrementa hasta adquirir rasgos de una economía capitalista.
La tendencia se representa en una $U$ inversa, expresando el ingreso per cápita con un indicador de desigualdad, en este caso el coeficiente de Gini. La figura 1, la hipótesis de Kuznets, describe las tendencias, en la curva representa la evolución histórica de los indicadores; entonces, una población donde todos son pobres existe igualdad de condiciones, cuando se incentiva el desarrollo económico, de una forma explosiva implica desigualdad y se llega hasta un punto crítico, donde se retorna al ajuste.

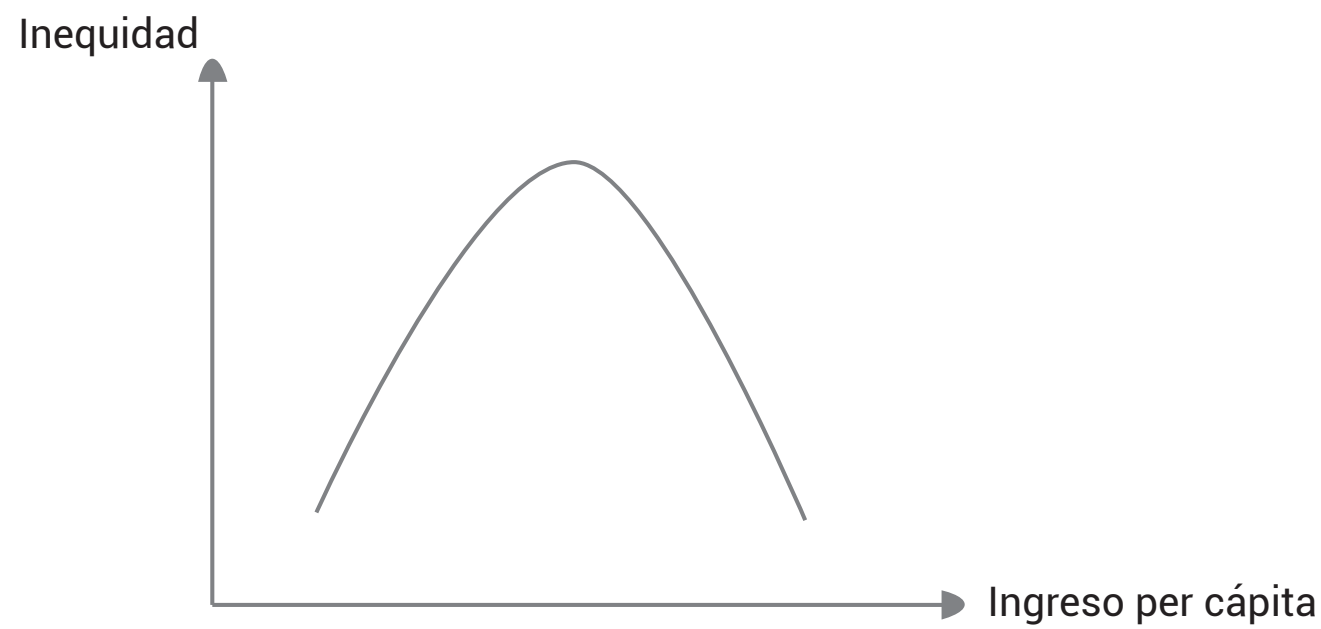

Figura 1. Curva de Kuznets.

Fuente: elaboración propia 


\section{INDICADOR DE DESIGUALDAD SOCIAL EN COLOMBIA}

Para el caso en Colombia, antes del año 2000, la pobreza se calculaba con la metodología de Necesidades Básicas Insatisfechas por esta razón no era posible realizar comparaciones. El índice de igualdad refleja mejores condiciones en los años siguientes, y muestra un incremento en la desigualdad en los años 2000 y 2007 , superiores a los demás periodos. De otro lado, el indicador de pobreza Índice de Pobreza Monetaria experimenta una reducción porcentual sostenida, incluso durante los siguientes 16 años, en el caso de la desigualdad a partir del año 2008 y hasta el 2016, que es el periodo de estudio, y mantiene una tendencia a reducir.

La primera década del presente siglo se caracterizó por un alto nivel de atención directa a la población en estado de pobreza como consecuencia de la generación de políticas públicas. Los resultados fueron netamente de necesidades económicas, se puede afirmar que fue un periodo de desarrollo económico y coincide con las características descritas por la hipótesis de Kuznets, donde la equidad social encuentra un punto crítico en el año 2007, los resultados obtenidos en el cálculo por Índice de Pobreza Multidimensional generaron respuesta durante los siguientes años, lo cual podría asegurar una estabilidad en el ingreso de las personas y efectivamente se traduce en un indicador sostenido en el coeficiente de Gini, en el período de 2008-2016.

Tabla 3. Evolución Indicador de Desigualdad en Colombia (1995-2016).

\begin{tabular}{|c|c|}
\hline Años & $\begin{array}{c}\text { Coeficiente } \\
\text { de Gini }\end{array}$ \\
\hline 1995 & 0,481 \\
\hline 1996 & 0,46 \\
\hline 1997 & 0,482 \\
\hline 1998 & 0,485 \\
\hline 1999 & 0,515 \\
\hline 2000 & 0,51 \\
\hline 2001 & 0,516 \\
\hline 2002 & 0,54 \\
\hline 2003 & 0,545 \\
\hline 2004 & 0,545 \\
\hline 2005 & 0,545 \\
\hline 2006 & 0,549 \\
\hline 2007 & 0,557 \\
\hline 2008 & 0,554 \\
\hline 2009 & 0,545 \\
\hline 2010 & 0,542 \\
\hline 2011 & 0,54 \\
\hline 2012 & 0,538 \\
\hline 2013 & 0,537 \\
\hline 2014 & 0,536 \\
\hline 2015 & 0,522 \\
\hline 2016 & 0,517 \\
\hline
\end{tabular}

Fuente: elaboración propia a partir de datos del DANE. 


\section{IMPACTO DE LA POLÍTICA SOCIAL EN LOS INDICADORES DEL ÍNDICE DE POBREZA MONETARIA Y EL INDICADOR DE EQUIDAD SOCIAL}

La figura 2 representa la evolución del indicador de equidad para Colombia en un periodo de veinte años (1995-2016) donde la hipótesis de Kuznets se comprueba. Esto se constata en la curva en $U$ inversa de una evolución que tiene un mínimo de inferencia, pero que finalmente es lo enunciado por Simón Kuznets. Se complementa con la figura 3 , donde se recrea la evolución del Índice de Pobreza Monetaria; y con la gráfica 4, el Índice de Pobreza Multidimensional, donde los tres indicadores se inclinan con el mismo objetivo durante ocho años consecutivos (2008-2016). Con estos resultados se confirma la incidencia positiva de la aplicación de políticas sociales - resultado de la medición por el IPM- sobre el indicador monetario y la tendencia positiva a la equidad social que está muy lejos de lograr niveles óptimos, pero se puede considerar un inicio a largo plazo de mejores resultados.

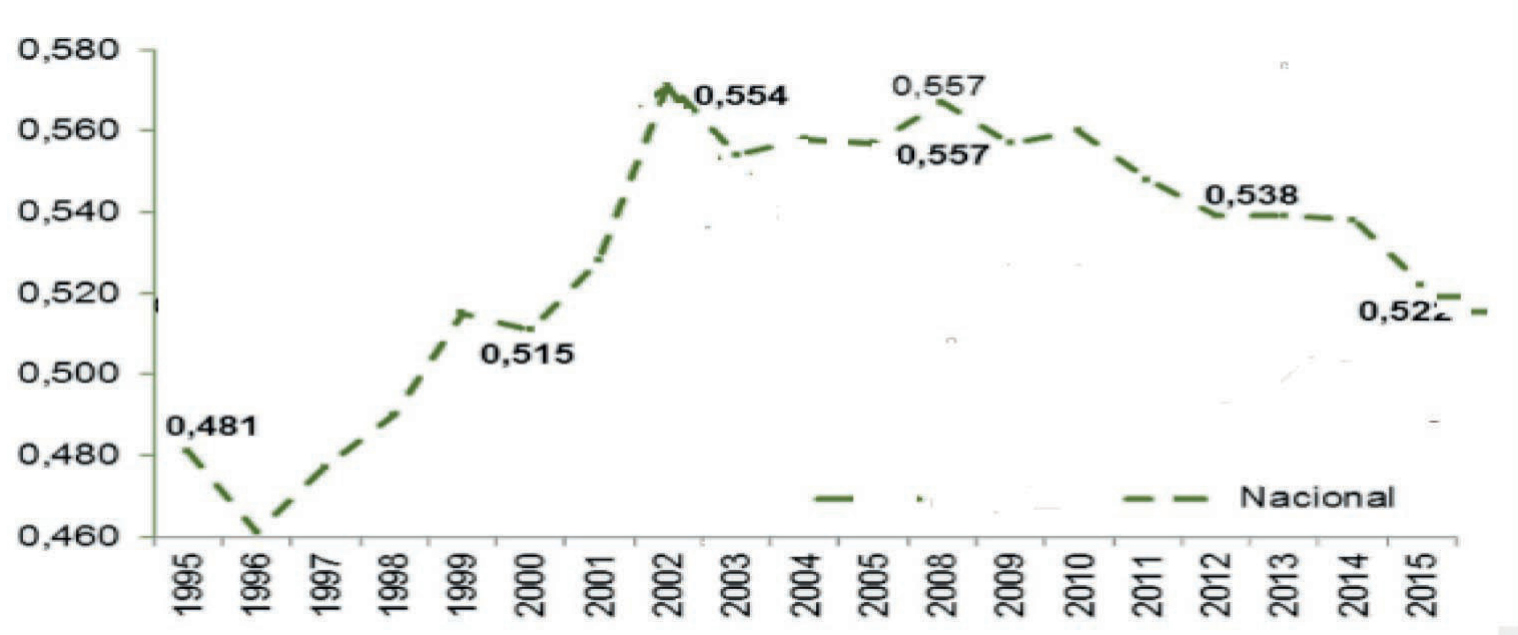

Figura 2. Coeficiente de Gini en Colombia 1996-2016.

Fuente: elaboración propia a partir de datos del DANE. 


\section{: Estrategia Organizacional}

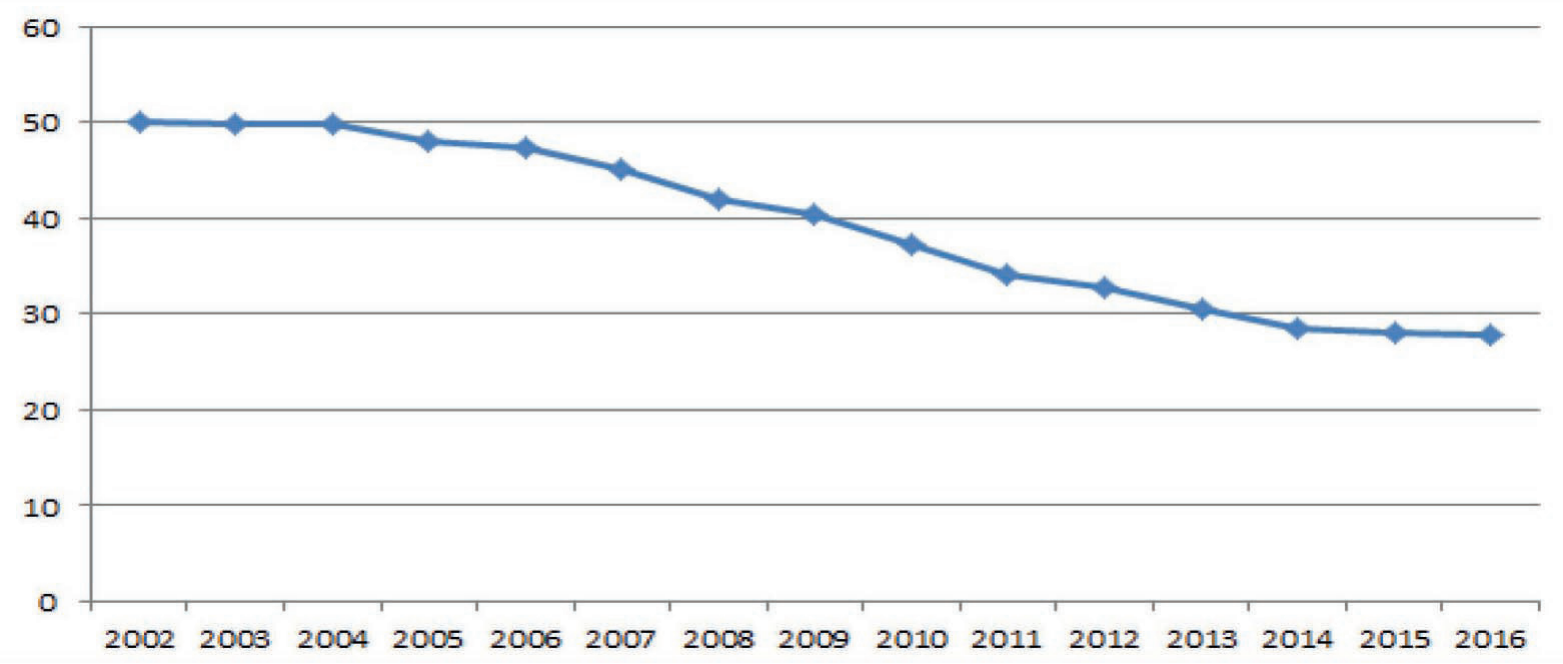

Figura 3. Índice de Pobreza Monetaria en Colombia 2002-2016.

Fuente: elaboración propia a partir de datos del DANE.

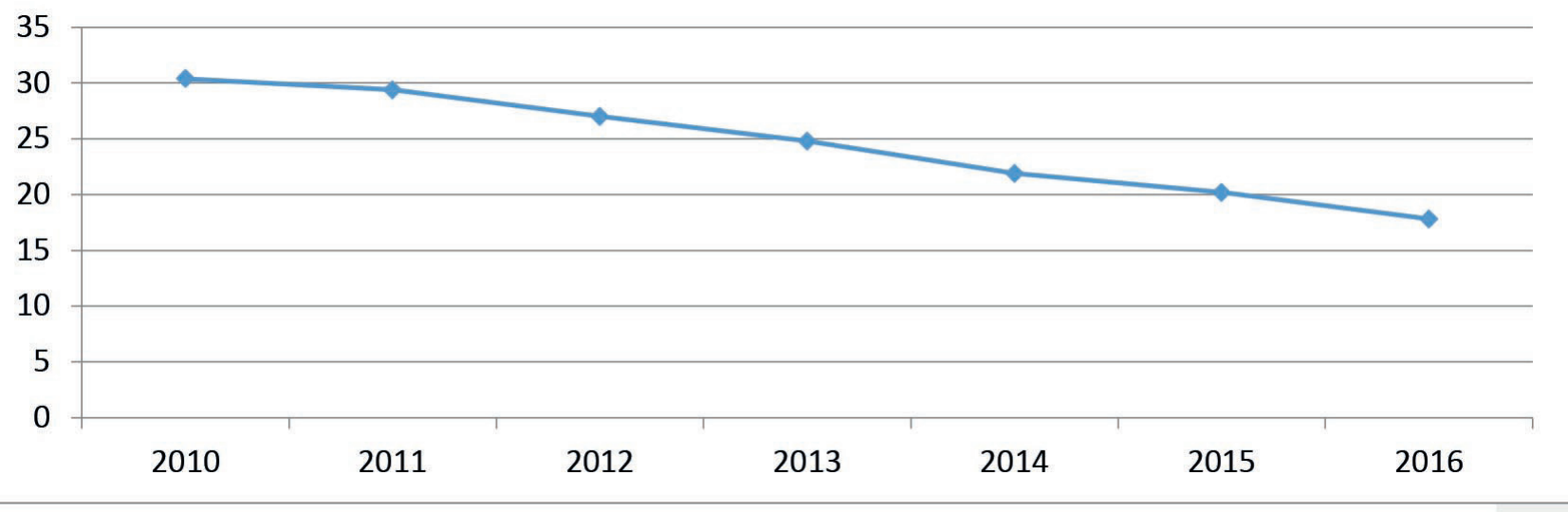

Figura 4. Índice de Pobreza Monetaria en Colombia 2002-2016.

Fuente: elaboración propia a partir de datos del DANE. 


\section{CONCLUSIONES}

Cuando se pretende encontrar equidad social en un pueblo es indispensable que se priorice el bienestar de los individuos, su formación y el desarrollo de las competencias necesarias para adaptar una vida en comunidad.

El desarrollo personal y colectivo de la humanidad, su crecimiento integral en busca de una verdadera calidad de vida genera por sí mismo el desarrollo económico y la estabilidad financiera de un país.

El enfoque de la medición con el Índice de Pobreza Multidimensional para Colombia ha permitido identificar la población con carencia de servicios, a quienes se les está negando la oportunidad de su desarrollo como seres humanos. Este punto de vista va más allá de ver la pobreza como una simple carencia de ingresos, la entiende como la negación para tener la posibilidad de realización de una vida con calidad aceptada por una sociedad culta.

El único camino para acercar los indicadores nacionales de pobreza a porcentajes inferiores o nulos, es sin duda la cobertura total en educación de la sociedad, una tarea imposible de obtener en el corto plazo. La intención del gobierno Colombiano, en el periodo citado, es obtener resultados a mediano y largo plazo y se refleja claramente en la concentración de recursos para la primera infancia, la creación de la Ley de Cero a Siempre y el despliegue de múltiples políticas enfocadas a la formación de las nuevas generaciones, tarea que requiere continuidad para el aseguramiento de los resultados.

Desde la perspectiva de gerencia en las entidades del Estado es imperativo entender que el ser humano es el beneficiario y debe ser enfocado como colectivo, y no tratarlo desde el punto de vista individual, porque las estructuras y modelos políticos no tienen razón de ser si el objeto no es el beneficio general.

La evolución de la sociedad no es un asunto de corto o mediano plazo, su efecto siempre muestra resultados en las generaciones futuras. En este sentido, la voluntad de enfocar las políticas sociales no debe depender sólo del gobierno de turno sino del impulso de toda la sociedad para construir una estructura democrática que exija los derechos generales por encima de pretensiones particulares.

El enfoque que debe priorizar las decisiones sociales por encima de las monetarias, ésta es una visión que sólo la academia puede procurar por investigar y ejercer un efecto en la sociedad, es la gran tarea de las ciencias sociales, la fusión de los resultados del conocimiento y la investigación en la aplicación del beneficio humano como colectivo, con el 
respeto a las demás especies y a los recursos naturales que afecten la calidad de vida de próximas generaciones.

La evolución general de reducción de la pobreza en Colombia por el IPM, desde nuestro análisis, es un proceso lento, pero permite el paso real de una sociedad pobre a una sociedad con posibilidades de desarrollo humano digno. Está en un segundo plano la necesidad directa económica, que sólo asegura el cambio de indicadores a corto plazo sin transcendencia en la formación de nuevas generaciones.

\section{REFERENCIAS}

Angulo, R. (2014). La década ganada: evolución de la clase media, la pobreza y la vulnerabilidad en Colombia 2002-2011. Coyuntura Económica, XLIV (1): 176.

Aguilar, L. (2013). Gobierno del Gobierno. Toluca, México: Instituto Nacional de Administración Publica.

Aparicio, A. (2009). Happiness and Growing Consumer Aspirations in Post-Modern Society. Revista Mexicana de Sociologia, 71 (1).

Barba Solano, C. (1995). La política social desde una perspectiva sociológica. Espiral, Estudios sobre Estado y Sociedad, II (4): 27-41.
Barreto Henríquez, M. (2014). Preparing for post-conflict in colombia from development and peace programs: challenges and learned lessons for international cooperation and companies. Revista Relaciones Internacionales, Estrategia y Seguridad, 9 (1): 179-197.

Bonilla Montenegro, J. D. (2015). Hydrocarbon extraction policy in Colombia and Ecuador: criticism from postdevelopment analysis. Analysis Politico, 83: 32-43.

Bucelly, J. F. (2015). Conditional cash transfer schemes and the politicisation of poverty reduction strategies. Analysis Politico, 83: 15-31.

Cañas, D. (2015). El origen socio-histórico de la normatividad científica. Revista Colombiana de Filosofía de la Ciencia, 15 (31): 55-82.

Carrizosa Umaña, J. (2015). El buen Vivir en Colombia. El Espectador. En línea. Recuperado de: https://www.elespectador.com/opinion/el-buenvivir-en-colombia-i-columna-561457

Carvajal, G. (2012). Sobre el discurso tecnológico de la modernidad. Revista Colombiana de Filosofía de la Ciencia, 12 (25): 37-60.

Delgado, R. (2007). Los marcos de acción colectiva y sus implicaciones culturales en la construcción de ciudadanía. Universitas Humanística, 64: 47. 
Fernández, M. (2000). La construcción social de la pobreza en la sociología de Simmel. Cuadernos de Trabajo Social, 13: 15-32.

Fajardo, G. (2015). Acceso efectivo a los servicios de salud: operacionalizando la cobertura universal en salud. Salud pública Mexicana, 57 (2): 3.

García, M.; Chicaiza, L. y Santos, A. (2016). Crisis financiera o de gestión, Evolución, del sector salud desde la perspectiva de los entes territoriales. Apuntes del Cenes, 35 (63): 77.

Leyva, S. (2014). Crisis or Continuity? A Discussion About Power in Medellin Since the 1970's. Revista Estudios Politicos, 44: 115-138

León, S. (2015). Formar ciudadanos desde el pre-escolar. Educación y Educadores, 18 (2): 250.

Martín, H. (2001). Viejas y nuevas formas de la ciudadanía. Revista de la CEPAL, 73: 117-128.

Macias, A. (2014). Crecimiento, Desigualdad y Pobreza. Revista de Economía Institucional, 16 (31): 101-126.

Ramírez, C. E. (2002). Pobreza en Colombia: tipos de medición y evolución de políticas entre los años 1950 y 2000 . Estudios Gerenciales, 85: 81-107.
Torres Guzmán, G. (2014). Criminality derived from natural disasters: proposal for the creation of public policy. Analysis Politico, 80: 53-78.

Vargas, I. (2010). Acceso a la atención en salud en Colombia. Rev. Salud Pública, 12 (5): 701-712.

Villaveces, J. (2009). Acción colectiva y el proceso de la política pública. Revista Opera, 9: 8.

Wallester, I. (2006). Abrir las ciencias sociales, Informe de la comisión Gulbenkian para la reestructuración de las ciencias sociales. México: Siglo XXI Editores S.A.

Zerda, Á. (2009). Mercado interno-Base dela acumulación productiva con equidad, Bases de una política económica para la productividad, el empleo y la distribución del ingreso. Bogotá, Colombia: Fundación Friederich Ebert de Colombia.

\section{DOCUMENTOS WEB}

Dimas Hoyos, D. (2016). En línea. Recuperado de: http://observatorio.desarrolloeconomico.gov. co/base/lectorpublic. php?id=838\#sthash. cOeqvZaj.dpbs.

Indicadores de pobreza y pobreza extrema utilizadas para el monitoreo de los ODM en América Latina. (2010). En línea. Recuperado de: http:// 
www.cepal.org/cgi-bin/getprod.asp? xml=/ MDG/noticias/paginas/1/40211/P40211. $\mathrm{xml} \& \mathrm{xs}=/ \mathrm{MDG} / \mathrm{tpl} / \mathrm{p} 18 \mathrm{f} . \mathrm{xs}$ |\&base=/MDG/tpl/ top-bottom.xsl.

Informe Acceso al cuidado en la primera Infancia. (2013). En línea. Recuperado de: http:// www.bogota.gov.co/article/estudio-muestra-que-el-acceso-al-cuidado-de-la-primera-infancia-aument\%C3\%B3-en-la-ciudad

Componentes y Estándares de Calidad en la educación inicial el marco de la atención integral, Ministerio de Educación Nacional. (2013). En línea. Recuperado de: http://www.mineducacion.gov.co/1759/w3-article-228881.html
Ley 1804 Establece La Política de Estado para el Desarrollo Integral de la Primera Infancia. (2016). En línea. Recuperado de: http://es.presidencia.gov.co/normativa/normativa/LEY\%20 $1804 \% 20$ DEL\%2002\%20DE\%20AGOSTO\%20 DE\%202016.pdf.

Atención Integral prosperidad para la primera Infancia. (2016). En línea. Recuperado de: http:// www.deceroasiempre.gov.co/QuienesSomos/ Documents / Cartilla-CerosiempreProsperidad-Primera-Infancia.pdf .

Las Bases de la Filosofía. (2013). Círculo de Viena. En línea. Recuperado de: http://lasbasesdelafilosofia.blogspot.com.co/p/circulo-de-viena.html. 